\title{
DOENÇAS CRÔNICAS E SONOLÊNCIA DIURNA EXCESSIVA EM PESSOAS IDOSAS
}

\section{Chronic diseases and excessive daytime sleepiness in older adults}

\section{Enfermedades crónicas y somnolencia diurna excesiva en mayores}

\author{
José Felipe Costa da Silva (OrcID) \\ Universidade Federal do Rio Grande do Norte - UFRN - Natal (RN) - Brasil
}

Edson Mendes Marques (OrclD)

Universidade Federal do Rio Grande do Norte - UFRN - Natal (RN) - Brasil

Thaiza Teixeira Xavier Nobre (OrcID)

Universidade Federal do Rio Grande do Norte - UFRN - Natal (RN) - Brasil

Isaac Newton Machado Bezerra (OrcID)

Universidade Federal do Rio Grande do Norte - UFRN - Natal (RN) - Brasil

Jônia Cybele Santos Lima (OrcID)

Universidade Federal do Rio Grande do Norte - UFRN - Natal (RN) - Brasil

\section{RESUMO}

Objetivo: Avaliar o nível de sonolência diurna excessiva (SDE), a síndrome de apneia obstrutiva do sono (SAOS) e as condições de saúde em idosos. Métodos: Trata-se de um estudo transversal e quantitativo, realizado com idosos cadastrados nas Estratégias de Saúde da Família de um município do Rio Grande do Norte, Brasil, de setembro de 2015 a janeiro de 2016. Utilizou-se um questionário sociodemográfico (com perguntas relativas ao: sexo, estado civil, tipo de moradia, endereço, renda, escolaridade e profissão) e de condições de saúde (com perguntas relativas à presença de doenças, a sequelas presentes e ao uso de medicamentos). A sonolência diurna excessiva (SDE) foi medida pela escala de Epworth e a síndrome de apneia obstrutiva do sono (SAOS), pelo questionário clínico de Berlin. Resultados: Participaram do estudo 61 idosos, com idade média de 68,5 anos, sendo 46 do sexo feminino $(75,4 \%)$. Dentre eles, $56(91,8 \%)$ relataram ter algum tipo de doença, sendo a hipertensão arterial sistêmica (HAS) a mais frequente $(57,4 \% ; n=35)$, seguida pela diabetes mellitus $(D M)(24,6 \% ; n=15)$ e artrite ou artrose (34,4\%; $n=21)$. Observou-se correlação positiva entre HAS e SAOS $(p=0,001)$, DM e SAOS ( $p=0,018)$ e entre SDE e doenças respiratórias $(p=0,010)$. Conclusão: As doenças mais prevalentes entre os idosos avaliados foram hipertensão arterial sistêmica e diabetes mellitus. A maioria não apresentou sonolência diurna excessiva e, quando observada, foi principalmente em grau leve. Apesar disso, observou-se associação positiva entre doenças crônicas, síndrome de apneia obstrutiva do sono e sonolência diurna excessiva.

Descritores: Distúrbios do Sono por Sonolência Excessiva; Atenção Primária à Saúde; Doença Crônica; Idoso.

\section{ABSTRACT}

Objective: To assess the level of Excessive Daytime Sleepiness (EDS), Obstructive Sleep Apnea Syndrome (OSAS) and health conditions in older adults. Methods: This is a quantitative cross-sectional study carried out with older adults enrolled in the Family Health Strategy in a municipality in Rio Grande do Norte, Brazil, from September 2015 to January 2016. A sociodemographic questionnaire (with questions related to: sex, marital status, type of housing, address, income, education and profession) and a health conditions questionnaire (with questions related to presence of diseases, sequelae and use of medications) were used. Excessive daytime sleepiness (EDS) was measured using the Epworth scale and sleep apnea obstructive syndrome (OSAS) was measured using the Berlin questionnaire. Results: Participants were 61 older adults with a mean age of 68.5 years, 46 of whom were women (75.4\%). In all, 56 (91.8\%) participants reported having some disease, with systemic arterial hypertension (SAH) being the most common (57.4\%; $n=35)$, followed by type 2 diabetes $(24.6 \%, n=15)$, and arthritis or arthrosis $(34.4 \%$, $n=21)$. There was a positive correlation between SAH and OSAS $(p=0.001), D M$ and OSAS $(p=0.018)$ and between EDS and respiratory diseases $(p=0.010)$. Conclusion: The most prevalent diseases in the older adults analyzed were systemic arterial hypertension and diabetes mellitus. The majority did not present excessive daytime sleepiness, and some presented very low 
levels of such condition. Despite that, there was a positive association of chronic diseases with obstructive sleep apnea syndrome and excessive daytime sleepiness.

Descriptors: Disorders of Excessive Somnolence; Primary Health Care; Chronic Disease; Aged.

\section{RESUMEN}

Objetivo: Evaluar el nivel de Somnolencia Diurna Excesiva (SDE), el Síndrome de Apnea Obstructiva del Sueño (SAOS) y las condiciones de salud de mayores. Métodos: Se trata de un estudio transversal y cuantitativo realizado con mayores inscritos en la Estrategia de Salud de la Familia de un municipio de Rio Grande do Norte, Brasil, entre septiembre de 2015 y enero de 2016. Se utilizó un cuestionario sociodemográfico (con preguntas sobre el sexo, el estado civil, el tipo de vivienda, la dirección, la renta, la escolaridad y la profesión) y de las condiciones de salud (con preguntas sobre la presencia de enfermedades, las secuelas y el uso de medicamentos). La Somnolencia Diurna Excesiva (SDE) fue medida por la escala de Epworth y el Síndrome de la Apnea Obstructiva del Sueño (SAOS) por el cuestionario de Berlin. Resultados: Participaron del estudio 61 mayores con media de edad de 68,5 años y 46 eran del sexo femenino (75,4\%). De entre ellos, $56(91,8 \%)$ relataron algún tipo de enfermedad y la hipertensión arterial sistémica (HAS) ha sido la más frecuente $(57,4 \% ; n=35)$ seguida por la diabetes mellitus $(D M)(24,6 \%$; $n=15)$ y artritis o artrosis (34,4\%; $n=21)$. Se observó una correlación positiva entre la HAS y el SAOS ( $p=0,001)$, la DM y el SAOS $(p=0,018)$ y entre la SDE y las enfermedades respiratorias $(p=0,010)$. Conclusión: Las enfermedades más frecuentes en los mayores evaluados fueron la hipertensión arterial sistémica y la diabetes mellitus. La mayoría no presentó somnolencia diurna excesiva y, si hubo, ha sido las de grado leve. A pesar de ello, se observó una asociación positiva entre las enfermedades crónicas, el síndrome de la apnea obstructiva del sueño y la somnolencia diurna excesiva.

Descriptores: Trastornos de Somnolencia Excesiva; Atención Primaria de Salud; Enfermedad Crónica; Anciano.

\section{INTRODUÇÃO}

O envelhecimento se caracteriza por um processo dinâmico que sofre influência de fatores biopsicossociais, ambientais e genéticos. Com a população envelhecendo, surge um elevado número de pessoas idosas no mundo e ocorre a diminuição da taxa de natalidade. e as melhorias nos sistemas socioeconômicos influenciaram nessa transição demográfica ${ }^{(1)}$. As mudanças epidemiológicas advindas dessa transição implicam também no aumento das doenças crônicas não transmissíveis (DCNT), já que os idosos são os principais afetados por esse conjunto de doenças e agravos. Esse processo se dá visto que o próprio processo de envelhecimento traz consigo vulnerabilidades limitantes ${ }^{(2)}$.

No ano de 2010, a principal causa de mortalidade no Brasil foram as doenças cardiovasculares ${ }^{(3)}$. Informações de levantamento do sistema brasileiro de Vigilância de Fatores de Risco e Proteção para Doenças Crônicas por Inquérito Telefônico (VIGITEL), realizado em 2016, mostram o avanço de condições favoráveis ao desenvolvimento de doenças crônicas, como o excesso de peso, que passou de 42,6\% em 2006 para 53,08\% em 2016. Além disso, os casos de obesidade apresentaram um grande aumento, passando de $11,8 \%$ para $18,9 \%$ dentro do mesmo período. O levantamento mostrou que, em uma década, o número de diabéticos aumentou $61,8 \%$, enquanto o número de hipertensos teve um aumento de $14,2 \%^{(4)}$. Outros fatores de risco, como obesidade, tabagismo e insuficiência de atividade física, influenciam negativamente a qualidade de vida dos indivíduos ${ }^{(5,6)}$.

Estudo realizado no Rio Grande do Norte, em 2014, apontou que as DCNT são as principais causas de morte entre a população idosa. Entre 2001 e 2011, 32,76\% das mortes entre idosos de 60 a 69 anos foram devido às doenças do aparelho circulatório; já entre os idosos de 80 anos ou mais, essa porcentagem atinge $35,26 \%$. Esse mesmo estudo apontou, ainda, um índice elevado de falecimentos por neoplasias ${ }^{(7)}$.

Dentre as principais DCNT, destaca-se a hipertensão arterial sistêmica (HAS), caracterizada por níveis elevados e sustentados de pressão arterial, que compromete órgãos como coração, encéfalo, rins e vasos sanguíneos ${ }^{(8)}$, e sua predominância ocorre na população idosa do sexo feminino e de baixa escolaridade ${ }^{(9)}$. Junto com as DCNT, é comum idosos relatarem problemas com o sono, impactando diretamente na sua qualidade de vida ${ }^{(5)}$.

Portanto, o sono é um dos aspectos que merece destaque na saúde do idoso, pois algumas de suas características sofrem mudança com o passar dos anos, havendo: a diminuição global do tempo de sono noturno; o aumento do tempo de latência de adormecimento; o avanço do ciclo circadiano, indo mais cedo para a cama e despertando de forma precoce durante a noite; a fragmentação do sono com várias interrupções e sonolência diurna. Na literatura, estudos demonstram que outros sinais e sintomas estão associados à sonolência, como tonturas e vertigens, que diminuem o desempenho funcional, aumentando significativamente o risco de quedas ${ }^{(10,11)}$. 
A sonolência diurna excessiva (SDE) sugere distúrbios e alterações do sono, sendo um forte sinal para apneia obstrutiva do sono, problema geralmente subdiagnosticado que aumenta os riscos cardiovasculares, a depressão e a obesidade, variando de acordo com idade e $\operatorname{sexo}^{(12-14)}$.

O uso contínuo de medicamentos pelos idosos com DCNT pode influenciar negativamente nos padrões e na qualidade do sono, sendo necessárias outras abordagens de tratamento não farmacológico, como a higiene do sono, que consiste em orientar mudanças de hábitos prejudiciais, promovendo a melhoria da qualidade do sono e saúde dessa população(15).

Percebendo o crescimento do grupo idoso no Brasil, o aumento das DCNT e a necessidade do conhecimento acerca da qualidade de sono dessa população, o objetivo deste trabalho é avaliar o nível de sonolência diurna excessiva, a síndrome de apneia obstrutiva do sono e as condições de saúde em idosos.

\section{MÉTODOS}

Trata-se de um estudo transversal, com abordagem quantitativa, desenvolvido no período de novembro de 2015 a janeiro de 2016, nas Estratégias de Saúde da Família (ESF) dos bairros Dner, Paraíso I e Cônego Monte do município de Santa Cruz, Rio Grande do Norte, Brasil. A amostra foi selecionada por conveniência e formada por idosos cadastrados nessas ESF.

Os usuários foram convidados a participar da pesquisa, sendo incluídos aqueles com idade de 60 anos ou mais, que apresentavam funções cognitivas preservadas ou comprometimento cognitivo leve, comprovado pelo escore abaixo do esperado no domínio memória imediata, de acordo com o nível de escolaridade do mini exame do estado mental ${ }^{(16)}$. Excluíram-se aqueles que se recusaram a responder aos instrumentos de avaliação ou que não apareceram nos dias marcados para as entrevistas.

A coleta de dados ocorreu através de um questionário semiestruturado, elaborado pelos pesquisadores responsáveis, com perguntas relacionadas a condições clínicas, como: presença de doenças, sequelas e uso de medicamentos, e questões sociodemográficas, com sexo, estado civil, tipo de moradia, endereço, renda, escolaridade e profissão. A escolaridade foi classificada como baixa (até fundamental completo), média (ensino médio completo) e alta (nível superior completo). As entrevistas ocorreram nas residências dos idosos e na sede da Faculdade de Ciências da Saúde do Trairi (FACISA).

Com o intuito de avaliar a SDE, foi utilizada a escala de sonolência de Epworth (ESE-BR), traduzida e validada para o Brasil(12). Trata-se de um questionário autoaplicável que avalia a probabilidade de adormecer em oito situações durante o dia: sentado e lendo; vendo televisão; sentado em lugares públicos; andando de trem, carro ou ônibus por uma hora, sem parar; deitando-se à tarde para descansar sentado e conversando com alguém; sentado calmamente, após o almoço, sem ter tomado bebida alcoólica; e sentado à direção de um carro parado, por alguns minutos, em trânsito intenso ${ }^{(12)}$.

O valor encontrado com a soma das oito situações diárias gera um escore entre 0 e 24 pontos, podendo classificar a sonolência do indivíduo em: de 0 a 9, limite; de 10 a 14, leve; de 15 a 20, moderada; e acima de 20, grave. Para a avaliação, o indivíduo deve fornecer uma nota de zero a três, quantificando sua tendência a: adormecer em nenhuma chance $=0$; pequena $=1$; moderada $=2$ e alta chance de cochilar $=3$. As respostas atingem valores máximos de 24 e mínimos de 0 pontos, sendo 10 o divisor da normalidade ${ }^{(12)}$.

Com o intuito de se investigar o risco para síndrome de apneia obstrutiva do sono (SAOS), que apresenta como um dos sintomas referidos a SDE, utilizou-se o questionário clínico de Berlin ${ }^{(17)}$ para verificar a presença da SAOS nos idosos portadores de diabetes tipo 2 e HAS.

A análise estatística descritiva simples ocorreu por meio do software Statistical Package for the Social Sciences (SPSS), versão 20.0, sendo apresentada através de tabelas com médias, medianas e desvio padrão para as variáveis quantitativas, e as variáveis categóricas foram expressas utilizando valores percentuais. Para verificar a correlação estatística entre HAS ou diabetes tipo 2 com a SAOS, utilizou-se o teste exato de Fisher. Para verificação de correlação entre doenças respiratórias com SDE, foi utilizado o teste de correlação de Pearson. Para ambas as associações, adotaram-se intervalos de confiança de $95 \%$, sendo estatisticamente significante o valor de $p<0,05$.

O presente trabalho recebeu aprovação pelo Comitê de Ética em Pesquisa da Universidade Federal do Rio Grande do Norte - UFRN (Parecer n. 1.116.397) e obedeceu às normas éticas exigidas pela Resolução $n^{\circ}$ 466/2016 (Conselho Nacional de Saúde). Após todo o esclarecimento acerca da pesquisa, os idosos que aceitaram participar assinaram o Termo de Consentimento Livre e Esclarecido (TCLE). 


\section{RESULTADOS}

A amostra foi composta por 61 idosos residentes em comunidades, com média de idade de $68,5( \pm 7,4)$ anos. Dos participantes, 46 eram do sexo feminino $(75,4 \%)$ e 15 eram do sexo masculino $(24,6 \%)$. Em relação ao estado civil, $52,5 \%(n=32)$ dos idosos eram casados e $29,5 \%(n=18)$ eram solteiros. Ao analisar a escolaridade $35(57,4 \%)$ eram analfabetos ou possuíam baixa escolaridade, e $26(42,6 \%)$ possuíam alta ou média escolaridade. Em relação à renda, $52(85,2 \%)$ relataram aposentadoria com fonte de renda. No que se refere ao tipo de moradia, $58(95,1 \%)$ moravam em casa própria e todos eram moradores da zona urbana. Questionados sobre suas ocupações, $19(31,1 \%)$ eram do lar, $16(26,2 \%)$ eram agricultores, $13(21,3 \%)$ eram autônomos, $8(13,1 \%)$ eram funcionários públicos e 5 $(8,2 \%)$ eram costureiras (Tabela I).

Na Tabela II, observam-se as condições de saúde da população estudada, em que 56 (91,8\%) possuíam algum tipo de doença. A HAS foi a mais prevalente, em 35(57,4\%) idosos, seguida pelo diabetes mellitus (DM) em 15 $(24,6 \%)$ e artrite ou artrose em $21(34,4)$.

Tabela I - Distribuição dos idosos segundo as variáveis sociodemográficas. Santa Cruz, Rio Grande do Norte, Brasil, 2016.

\begin{tabular}{|c|c|c|}
\hline Características & $\mathbf{n}$ & $\%$ \\
\hline \multicolumn{3}{|l|}{ Sexo } \\
\hline Feminino & 46 & 75,4 \\
\hline Masculino & 15 & 24,6 \\
\hline \multicolumn{3}{|l|}{ Estado civil } \\
\hline Casado & 32 & 52,5 \\
\hline Solteiro & 18 & 29,5 \\
\hline Viúvo & 7 & 11,5 \\
\hline Separado & 4 & 6,6 \\
\hline \multicolumn{3}{|l|}{ Escolaridade } \\
\hline Analfabeto e baixa escolaridade & 35 & 57,4 \\
\hline Média e alta escolaridade & 26 & 42,6 \\
\hline \multicolumn{3}{|l|}{ Renda } \\
\hline Aposentadoria & 52 & 85,2 \\
\hline Aposentadoria + pensão & 9 & 14,8 \\
\hline \multicolumn{3}{|l|}{ Tipo de moradia } \\
\hline Própria & 58 & 95,1 \\
\hline Alugada & 3 & 4,9 \\
\hline \multicolumn{3}{|l|}{ Local de moradia } \\
\hline Zona urbana & 61 & 100,0 \\
\hline Zona rural & 0 & 0,0 \\
\hline \multicolumn{3}{|l|}{ Profissão } \\
\hline Do lar & 19 & 31,1 \\
\hline Agricultor & 16 & 26,2 \\
\hline Autônomo & 13 & 21,3 \\
\hline Funcionário público & 8 & 13,1 \\
\hline Costureira & 5 & 8,2 \\
\hline Total & 61 & 100,0 \\
\hline
\end{tabular}


Tabela II - Distribuição dos idosos segundo as condições de saúde. Santa Cruz, Rio Grande do Norte, Brasil, 2016.

\begin{tabular}{|c|c|c|}
\hline Características & $\mathbf{n}$ & $\%$ \\
\hline \multicolumn{3}{|l|}{ Doença } \\
\hline Sim & 56 & 91,8 \\
\hline Não & 5 & 8,2 \\
\hline \multicolumn{3}{|c|}{ Hipertensão arterial sistêmica } \\
\hline Sim & 35 & 57,4 \\
\hline Não & 26 & 42,6 \\
\hline \multicolumn{3}{|l|}{ Diabetes Mellitus } \\
\hline Sim & 15 & 24,6 \\
\hline Não & 46 & 75,4 \\
\hline \multicolumn{3}{|l|}{ Atrite ou artrose } \\
\hline Sim & 21 & 34,4 \\
\hline Não & 40 & 65,6 \\
\hline \multicolumn{3}{|l|}{ Depressão } \\
\hline Sim & 12 & 19,7 \\
\hline Não & 49 & 80,3 \\
\hline \multicolumn{3}{|c|}{ Doenças respiratórias } \\
\hline Sim & 5 & 8,2 \\
\hline Não & 56 & 91,8 \\
\hline \multicolumn{3}{|l|}{ Osteoporose } \\
\hline Sim & 3 & 4,9 \\
\hline Não & 58 & 95,1 \\
\hline \multicolumn{3}{|c|}{ Doenças cardíacas } \\
\hline Sim & 3 & 4,9 \\
\hline Não & 58 & 95,1 \\
\hline \multicolumn{3}{|c|}{ Autopercepção de saúde } \\
\hline Muito boa & 2 & 3,3 \\
\hline Boa & 27 & 44,3 \\
\hline Mais ou menos & 28 & 45,9 \\
\hline Ruim & 2 & 3,3 \\
\hline Não sei & 2 & 3,3 \\
\hline Total & 61 & 100,0 \\
\hline
\end{tabular}

Na Tabela III, encontram-se os dados da avaliação da sonolência diurna excessiva através da escala de sonolência de Epworth (ESE-BR). Observa-se que 36 (59\%) idosos foram classificados como normal, 17 (27,9\%) apresentaram grau leve de sonolência diurna excessiva, $7(11,5 \%)$ apresentaram grau moderado e $1(1,6 \%)$ apresentou grau grave.

Observou-se correlação positiva entre a presença de HAS e SAOS $(p=0,001)$ e também correlação positiva entre DM e SAOS $(p=0,018)$. Além disso, a correlação entre SDE e doenças respiratórias foi significativa através da correlação de Pearson $(\mathrm{p}=0,010)$. As demais variáveis não apresentaram valor significante de correlação (Tabela IV).

Tabela III - Distribuição dos idosos segundo a Escala de sonolência de Epworth (ESE-BR). Santa Cruz, Rio Grande do Norte, Brasil, 2016.

\begin{tabular}{lcc}
\hline Escala de sonolência de Epworth & $\mathbf{n}$ & \% \\
\hline Normal & 36 & 59,0 \\
Leve & 17 & 27,9 \\
Moderada & 7 & 11,5 \\
Grave & 1 & 1,6 \\
Total & 61 & 100,0 \\
\hline
\end{tabular}

Tabela IV - Associação de doenças crônicas e distúrbios do sono em idosos. Santa Cruz, Rio Grande do Norte, Brasil, 2016.

\begin{tabular}{lcc}
\hline Doença & Avaliação do sono & valor de $\mathbf{p}$ \\
\hline Doenças respiratórias & SAOS & $0,010^{*}$ \\
Hipertensão arterial sistêmica & SDE & $0,001^{* *}$ \\
Diabetes mellitus & SDE & $0,018^{* *}$ \\
\hline
\end{tabular}

"correlação de Pearson; "* Teste Exato de Fisher, SAOS: Síndrome de apneia obstrutiva do sono; SDE: Sonolência diurna excessiva 


\section{DISCUSSÃO}

Dos 61 idosos avaliados na presente pesquisa, a maioria era do sexo feminino, casado, com baixa escolaridade, moravam em casa própria e tinham como principal fonte de renda a aposentadoria. As doenças mais prevalentes entre os idosos foram HAS e diabetes mellitus. Além disso, a maioria não apresentou SDE e, quando observada, foi principalmente no grau mais leve. Apesar disso, observou-se associação positiva entre doenças crônicas e distúrbios do sono, como a SDE e a SAOS, que apontam para a necessidade de se buscar medidas protetivas para a saúde desses idosos. Pesquisas apontam que a má qualidade do sono diminui a capacidade física e aumenta o risco de quedas em idosos, bem como diminuem as habilidades cognitivas ${ }^{(18,19)}$.

No Brasil, no ano de 2006, foi lançada a Política Nacional de Saúde da Pessoa Idosa, com o objetivo de melhorar as ações de saúde e acesso para essa população em ascensão. Além de outros objetivos, essa política pretendia investigar as necessidades básicas de saúde do idoso para uma boa qualidade de vida(20).

No atual trabalho foi percebido um maior percentual de mulheres na amostra, o que corrobora um estudo que mostra o aumento de expectativas de vida do sexo feminino e o processo de feminização do envelhecimento. Alguns fatores contribuem para esses resultados, como menor exposição a fatores de risco, maior incentivo de políticas públicas de saúde da mulher, e barreiras sociais e culturais no acesso dos homens aos sistemas de saúde(21).

Dentre as doenças mais prevalentes, a HAS apresentou destaque como a mais relatada pelos idosos da atual pesquisa. Além disso, houve correlação positiva entre a presença de HAS e SAOS $(p=0,001)$. A HAS é uma das afecções mais comuns na população idosa, no entanto ainda existe uma grande parcela dos portadores que não são acompanhados, aumentando os riscos de agravantes ${ }^{(22)}$. Além do mais, ela faz parte das doenças cardiovasculares com origem multifatorial, sendo a principal causadora de morbidade e mortalidade no mundo. Na literatura já foi mostrada correlação entre distúrbios do sono, morte súbita e HAS. Portanto, a determinação dos fatores de risco contribui para a prevenção, visto que podem ser minimizados com tratamento adequado(23).

Além da HAS, a diabetes mellitus foi a segunda condição mais prevalente entre os idosos avaliados na pesquisa em questão, sendo observada correlação positiva em DM e SAOS $(p=0,018)$. Em quatro anos, houve uma alta taxa de mortalidade por complicações agudas do DM no Norte e Nordeste do Brasil( ${ }^{(24)}$, e fatores como sexo masculino e HAS são preditores clínicos entre pacientes com diabetes tipo 2 para o surgimento de problemas relacionados ao sono ${ }^{(25)}$.

O sono é um processo biológico complexo e que apresenta mudanças intercaladas com a vigília e condições moduladas por hormônios. Esses processos alteram a temperatura corporal, o trabalho cardíaco e a produção hormonal, sendo um estado neurológico restaurador primordial para o bom funcionamento do organismo ${ }^{(26)}$. Desse modo, os distúrbios do sono são responsáveis por agravar outras condições clínicas nessa população, havendo potencialização da depressão e incapacidade funcional. Além disso, idosos que apresentam diminuição do tempo de sono ou de sua latência tendem a avaliar sua saúde de forma negativa(27).

As comorbidades estudadas no presente estudo também apresentam interação entre si, já que a sonolência diurna excessiva deve servir de alerta clínico para possíveis outras etiologias, como a narcolepsia, o uso errôneo de medicamentos e a apneia obstrutiva do sono ${ }^{(28)}$.

A SDE é a segunda queixa mais referida por indivíduos que procuram os laboratórios de sono ${ }^{(29)}$. No presente estudo, a associação entre SDE e doenças respiratórias foi significativa $(p=0,010)$, e entre as principais doenças respiratórias citadas na pesquisa está a asma. Na literatura, existem associações negativas entre asma e SDE e qualidade de vida, e a polimedicação e a presença de comorbidades associadas, geralmente comum nos idosos, agravam os problemas existentes de distúrbios do sono ${ }^{(30)}$.

O predomínio da SDE em idosos usuários da atenção primária está intimamente ligada à depressão, à obesidade e à percepção de saúde ruim ${ }^{(31)}$. Na avaliação de um estudo, percebeu-se que uma grande parcela estava em seus graus de normalidade, apesar dos estudos mostrarem que pacientes com idade igual ou superior a 60 anos, separados e índice de massa corporal superior a 30 apresentam maiores chances de referir sonolência ${ }^{(12)}$.

Os graus de SDE leve seguido por moderado foram os mais recorrentes na amostra da presente investigação. Um estudo realizado em São Paulo, no ano de 2015, já demostrava que pessoas do sexo feminino e com baixa escolaridade estão mais expostas a sofrerem com a SDE ${ }^{(32)}$, e seus sintomas estão sempre associados a distúrbios neurológicos e clínicos ${ }^{(3)}$. Doenças como DM, estados de ansiedade e depressão e uso crônico de tabaco aumentam as chances de o indivíduo sofrer com SDE. Esse processo desencadeia consequências graves à saúde e ao bemestar, afetando todas as áreas do indivíduo, com repercussões no emprego, nos relacionamentos e na cognição(34,35). 
Com base nos achados do atual estudo, faz-se necessária a realização de mais estudos que evidenciem outros problemas, relacionando o sono aos seus impactos na saúde das pessoas idosas na atenção primária, tendo em vista que esse é o principal ambiente para promoção à saúde da população. No mais, medidas de promoção à saúde dos idosos necessitam ser adotadas. A prática de exercícios físicos é apontada como fator de proteção, sendo capaz de diminuir os efeitos do envelhecimento, preservar as funções cognitivas e prolongar a autonomia dos idosos ${ }^{(36,37)}$.

Os questionários utilizados no presente estudo são importantes pela facilidade de aplicação por profissionais de saúde na população, contribuindo com a avaliação de queixas do sono, de maneira que podem ser instrumento para avaliação inicial do sono, triando os distúrbios do sono mais comuns e, com isso, auxiliando na construção de estratégias de combate, como higiene do sono, promovendo, a saúde da população idosa. Algumas limitações foram encontradas, como: número reduzido de idosos; o uso de poucos questionários e escalas para avaliar o sono; e a falta da polissonografia, o padrão ouro na avaliação do sono. Entretanto, apontam para o diagnóstico e alerta para distúrbios do sono e contribuem para a promoção da saúde da população investigada.

\section{CONCLUSÃO}

As doenças mais prevalentes entre os idosos avaliados foram hipertensão arterial sistêmica e diabetes mellitus. A maioria não apresentou sonolência diurna excessiva e, quando observada, foi principalmente em grau leve. Apesar disso, observou-se associação positiva entre doenças crônicas, síndrome de apneia obstrutiva do sono e sonolência diurna excessiva.

\section{CONTRIBUIÇÕES}

José Felipe Costa da Silva e Thaiza Teixeira Xavier Nobre contribuíram com a elaboração e delineamento do estudo; a aquisição, análise e interpretação dos dados; e com a redação e revisão do manuscrito. Edson Mendes Marques contribuiu com a redação e revisão do manuscrito. Isaac Newton Machado Bezerra contribuiu com a redação e revisão do manuscrito e a análise dos dados. Jônia Cybele Santos Lima contribuiu com a revisão do manuscrito e a análise e interpretação dos dados.

\section{CONFLITOS DE INTERESSE}

Os autores do presente artigo declaram não possuir conflitos de interesse, seja de ordem financeira, comercial, política, acadêmica ou pessoal.

\section{REFERÊNCIAS}

1. Silva DC, Antunes DZ. Dependência do idoso na execução das atividades de vida diária. Fasem Ciências [Internet]. 2014 [acesso em 2018 Set 23];3(1):41-54. Disponível em: http://www.fasem.edu.br/revista/index.php/ fasemciencias/article/view/55/pdf

2. Porciúncula R de $\mathrm{CR}$ da, Carvalho EF de, Barreto KML, Leite VMM. Perfil socioepidemiológico e autonomia de longevos em Recife-PE, Nordeste do Brasil. Rev Bras Geriatr Gerontol [Internet]. 2014 [acesso em 2018 Set 23];17(2):315-25. Disponível em: http://www.scielo.br/scielo.php?script=sci_arttext\&pid=S180998232014000200315\&lng=pt\&tlng=pt

3. Oliveira BHD, Yassuda MS, Cupertino APFB, Neri AL. Relações entre padrão do sono, saúde percebida e variáveis socioeconômicas em uma amostra de idosos residentes na comunidade: Estudo PENSA. Ciênc Saúde Coletiva [Internet]. 2010 [acesso em 2018 Set 23];15(3):851-60. Disponível em: http://www.scielo.br/scielo. php?script=sci_arttext\&pid=S1413-81232010000300028\&lng=pt\&nrm=iso\&tlng=pt

4. Ministério da Saúde (BR), Secretaria de Vigilância em Saúde, Departamento de Vigilância de Doenças e Agravos não Transmissíveis e Promoção da Saúde. Vigitel Brasil 2016: vigilância de fatores de risco e proteção para doenças crônicas por inquérito telefônico : estimativas sobre frequência e distribuição sociodemográfica de fatores de risco e proteção para doenças crônicas nas capitais dos 26 estados brasileiros e no Distrito Federal em 2016. Brasília: Ministério da Saúde; 2017.

5. Silva A, Prá KRD. Envelhecimento populacional no Brasil: elementos para pensar o lugar das famílias na proteção aos idosos. Argumentum. 2014;6(1):99-115. 
6. Monteiro NT, Ceolim MF. Quality of sleep of the aged at home and in hospital. Texto \& Contexto Enferm [Internet]. 2014 [acesso em 2018 Set 23];23(2):356-64. Disponível em: http://www.scielo.br/scielo.php?script=sci_ arttext\&pid=S0104-07072014000200356\&lng=en\&tIng=en

7. Oliveira TC, Medeiros WR, Lima KC. Diferenciais de mortalidade por causas nas faixas etárias limítrofes de idosos. Rev Bras Geriatr Gerontol [Internet]. 2015 [acesso em 2018 Set 23];18(1):85-94. Disponível em: http:// www.scielo.br/scielo.php?script=sci_arttext\&pid=S1809-98232015000100085\&lng=pt\&tlng=pt

8. Beserra A A, Silva LV L, Miranda ES, Almeida MR de, Santos SC. Conhecimento sobre fatores de risco para hipertensão arterial sistêmica por discentes do curso de enfermagem. Rev Bras Educ e Saúde [Internet]. 2017 [acesso em 2018 Set 23];7(2):61-7. Disponível em: http://www.gvaa.com.br/revista/index.php/REBES/article/ view/4217

9. Mendes GS, Moraes CF, Gomes L. Prevalência de hipertensão arterial sistêmica em idosos no Brasil entre 2006 e 2010. Rev Bras Med Família e Comunidade [Internet]. 2014 [acesso em 2018 Set 23];9(32):273-8. Disponível em: http://rbmfc.org.br/rbmfc/article/view/795

10. Menon-Miyake MA, Santana GG, Menon-Miyake M, Menon-Miyake M. Distúrbios do sono e sintomas vestibulares. Rev Equilíbrio Corporal Saúde 2014;6(2):60-6.

11. Pereira AA, Neri AL, Ceolim MF. Association between insomnia symptoms, daytime napping, and falls in communitydwelling elderly. Cad Saúde Pública [Internet]. 2013 [acesso em 2018 Set 23];29(3):535-46. Disponível em: http://www.ncbi.nlm.nih.gov/pubmed/23532288

12. Andrechuk CRS, Ceolim MF. Sonolência diurna excessiva nos pacientes com infarto agudo do miocárdio. Acta Paul Enferm [Internet]. 2015 [acesso em 2018 Set 23];28(3):230-6. Disponível em: http://dx.doi.org/10.1590/1982-

13. Lopes JM, Dantas FG, Medeiros JLA. Excessive daytime sleepiness in the elderly: association with cardiovascular risk, obesity and depression. Rev Bras Epidemiol [Internet]. 2013 [acesso em 2018 Set 23];16(4):872-9. Disponível em: http://www.scielo.br/scielo.php?script=sci_arttext\&pid=S1415-790X2013000400872\&Ing=en\&tlng=en

14. Campostrini D, Prado L, Prado G. Síndrome da apneia obstrutiva do sono e doenças cardiovasculares. Rev Neurociências [Internet]. 2014 [acesso em 2018 Set 23];22(1):102-12. Disponível em: http://www. revistaneurociencias.com.br/edicoes/2014/2201/2201revisao/930revisao.pdf

15. Corrêa CC, Campos LD, Weber SAT. Conscientização sobre hábitos relacionados ao sono no interior do estado de São Paulo. Arch Heal Investig [Internet]. 2018 [acesso em 2018 Set 23];7(3):115-7. Disponível em: http:// archhealthinvestigation.com.br/ArcHI/article/view/2810

16. Bertolucci PHF, Brucki SMD, Campacci SR, Juliano Y. O mini-exame do estado mental em uma população geral: impacto da escolaridade. Arq Neuropsiquiatr [Internet]. 1994 [acesso em 2018 Set 23];52(1):1-7. Disponível em: http://www.scielo.br/scielo.php?script=sci_arttext\&pid=S0004-282X1994000100001\&lng=pt\&tlng=pt

17. Araújo-Melo MH, Neves DD, Ferreira LVMV, Moreira MLV, Nigri R, Simões SMG. Questionários e Escalas úteis na pesquisa da Síndrome da Apneia Obstrutiva do Sono. Rev Hosp Univ Pedro Ernesto [Internet]. 2016 [acesso em 2018 Set 23];15(1):48-54. Disponível em: http://www.e-publicacoes.uerj.br/index.php/revistahupe/article/ view/22368

18. Cruz DT, Cruz FM, Ribeiro AL, Veiga CL, Leite ICG. Associação entre capacidade cognitiva e ocorrência de quedas em idosos. Cad Saúde Coletiva [Internet]. 2015 [acesso em 2018 Set 23];23(4):386-93. Disponível em: http://www.scielo.br/scielo.php?script=sci_arttext\&pid=S1414-462X2015000400386\&lng=pt\&tlng=pt

19. Ferretti F, Castanha AC, Padoan ER, Lutinski J, Silva MR. Quality of life in the elderly with and without chronic pain. Brazilian J Pain [Internet]. 2018 [acesso em 2018 Set 23];1(2):141-6. Disponível em: http://www.gnresearch. org/doi/10.5935/2595-0118.20180022

20. Brasil. Ministério da Saúde. Política Nacional de Saúde da Pessoa Idosa. Portaria $n^{\circ} 2.529 / G M$ de 19 de outubro de 2006. Institui a Internação Domiciliar no âmbito do SUS. Diário Oficial da União; Brasília; 20 out. 2006 [acesso em 2018 Set 23]. Disponível em: http://bvsms.saude.gov.br/bvs/saudelegis/gm/2006/prt2528_19_10_2006.html

21. Pimenta FB, Pinho L, Silveira MF, Botelho ACC. Fatores associados a doenças crônicas em idosos atendidos pela Estratégia de Saúde da Família. Ciênc Saúde Coletiva [Internet]. 2015 [acesso em 2018 Set 23];20(8):2489- 
98. Disponível em: http://www.scielo.br/scielo.php?script=sci_arttext\&pid=S1413-81232015000802489\&Ing=pt \&tlng=pt

22. Ferreira RA, Barreto SM, Giatti L. Hipertensão arterial referida e utilização de medicamentos de uso contínuo no Brasil: um estudo de base populacional. Cad Saúde Pública [Internet]. 2014 [acesso em 2018 Set 23];30(4):81526. Disponível em: http://www.scielo.br/scielo.php?script=sci_arttext\&pid=S0102-311X2014000400815\&lng=pt \&tlng=pt

23. Campostrini D, Prado L, Prado G. Síndrome da Apneia Obstrutiva do Sono e Doenças Cardiovasculares. Rev Neurociências [Internet]. 2014 [acesso em 2018 Set 23];22(1):102-12. Disponível em: http://www. revistaneurociencias.com.br/edicoes/2014/2201/2201revisao/930revisao.pdf

24. Klafke A, Duncan BB, Rosa RS, Moura L, Malta DC, Schmidt MI. Mortalidade por complicações agudas do diabetes melito no Brasil, 2006-2010. Epidemiol Serv Saúde [Internet]. 2014 [acesso em 2018 Set 23];23(3):45562. Disponível em: http://scielo.iec.pa.gov.br/scielo.php?script=sci_arttext\&pid=S1679-49742014000300008\&ln $\mathrm{g}=$ en\&nrm=iso\&tlng=en

25. Correia MABB, Souza MJMC, Oliveira RAP, Carvalho MMB, Medeiros AKL, Medeiros CA, et al. Preditores clínicos da apneia obstrutiva do sono entre diabéticos tipo 2. Sleep Sci [Internet]. 2015 [acesso em 2018 Set 23];8(4):221-2. Disponível em: https://www.sciencedirect.com/science/article/pii/S1984006316001103

26. Luyster FS, Strollo PJ, Zee PC, Walsh JK. Sleep: a health imperative. Sleep [Internet]. 2012 [acesso em 2018 Set 23];35(6):727-34. Disponível em: https://academic.oup.com/sleep/article-lookup/doi/10.5665/sleep.1846

27. Zawisza K, Tobiasz-Adamczyk B, Galas A, Brzyska M. Sleep duration and mortality among older adults in a 22-year follow-up study: an analysis of possible effect modifiers. Eur J Ageing. 2015;12(2):119-29.

28. Plazzi G, Ferri R, Antelmi E, Bayard S, Franceschini C, Cosentino FI, et al. Restless legs syndrome is frequent in narcolepsy with cataplexy patients. Sleep. 2010;33(5):689-94.

29. Aguiar IDC, Dias IS, Oliveira AR, Maria L, Sampaio M, Nacif SR, et al. Características Clínicas, funcionais e variáveis polissonográficas de pacientes de um laboratório de pesquisa em distúrbios do sono. J Health Sci (Londrina). 2011;13(4):227-32.

30. Marques IR, Isabel RDO. Avaliação da qualidade do sono e da qualidade de vida na asma. Rev Bras Qual Vida [Internet]. 2011 [acesso em 2018 Set 23];3(1):24-31. Disponível em: https://periodicos.utfpr.edu.br/rbqv/article/ view/757

31. Lima CA, Soares WJ de S, Bilton TL, Dias RC, Ferrioll E, Perracini MR. Correlates of excessive daytime sleepiness in community-dwelling older adults: an exploratory study. Rev Bras Epidemiol [Internet]. 2015 [acesso em 2018 Set 23];18(3):607-17. Disponível em: http://www.scielo.br/scielo.php?script=sci_arttext\&pid=S1415-790X2015 $000300607 \&$ lng $=$ en\&tlng=en

32. Zanuto EAC, Lima MCS, Araújo RG, Silva EP, Anzolin CC, Araujo MYC, et al. Distúrbios do sono em adultos de uma cidade do Estado de São Paulo. Rev Bras Epidemiol [Internet]. 2015 [acesso em 2018 Set 23];18(1):42-53. Disponível em: http://www.scielo.br/scielo.php?script=sci_arttext\&pid=S1415-790X2015000100042\&lng=pt\&tln $\mathrm{g}=\mathrm{pt}$

33. Giorelli AS, Santos PP, Carnaval T, Gomes MM. Sonolência excessiva diurna: aspectos clínicos, diagnóticos e terapêuticos. Rev Bras Neurol. 2012;48(3):17-24.

34. Medeiros C, Bruin V, Férrer D, Paiva T, Montenegro R Júnior, Forti A, et al. Excessive daytime sleepiness in type 2 diabetes. Arq Bras Endocrinol Metabol [Internet]. 2013 [acesso em 2018 Set 23];57(6):425-30. Disponível em: http://www.scielo.br/scielo.php?script=sci_arttext\&pid=S0004-27302013000600003\&lng=en\&tlng=en

35. Theorell-Haglöw J, Åkerstedt T, Schwarz J, Lindberg E. Predictors for Development of Excessive Daytime Sleepiness in Women: A Population-Based 10-Year Follow-Up. Sleep [Internet]. 2015 [acesso em 2018 Set 23];38(12):1995-2003. Disponível em: https://academic.oup.com/sleep/article-lookup/doi/10.5665/sleep.5258

36. Vigorito C, Giallauria F. Effects of exercise on cardiovascular performance in the elderly. Front Physiol [Internet]. 2014 [acesso em 2018 Set 23]. Disponível em: http://journal.frontiersin.org/article/10.3389/fphys.2014.00051/ abstract 
37. Camões M, Fernandes F, Silva B, Rodrigues T, Costa N, Bezerra P. Exercício físico e qualidade de vida em idosos: diferentes contextos sociocomportamentais. Motricidade [Internet]. 2016 [acesso em 2018 Set 23];12(1):96-105. Disponível em: http://revistas.rcaap.pt/motricidade/article/view/6301

\section{Endereço para correspondência:}

José Felipe Costa da Silva

Universidade Federal do Rio Grande do Norte - UFRN

Av. Senador Salgado Filho, 3000

Bairro: Lagoa Nova

CEP: 59078-970 - Natal - RN - Brasil

E-mail: felipedoshalom@yahoo.com.br 